\title{
Características histomorfométricas do intestino de juvenis de tambaqui após uso de probiótico na dieta e durante transporte ${ }^{1}$
}

\begin{abstract}
Celma M. Ferreira' ${ }^{2}$ Nadia A.B. Antoniassi ${ }^{3}$, Felipe G. Silva ${ }^{3}$, Jayme A. Povh ${ }^{4}$, Alexandra Potença ${ }^{3}$, Thayssa C.H. Moraes ${ }^{3}$, Thais K.S.T. Silva ${ }^{5}$ e Janessa S. Abreu ${ }^{3 *}$

ABSTRACT.- Ferreira C.M., Antoniassi N.A.B., Silva F.G., Povh J.A., Potença A., Moraes T.C.H., Silva T.K.S.T. \& Abreu J.S. 2014. [Histomorphometric characteristics gut of tambaqui after using probiotic on diet and during transport.] Características histomorfométricas do intestino de juvenis de tambaqui após uso de probiótico na dieta e durante transporte. Pesquisa Veterinária Brasileira 34(12):1258-1264. Faculdade de Agronomia, Medicina Veterinária e Zootecnia, Universidade Federal de Mato Grosso, Av. Fernando Corrêa da Costa 2367, Boa Esperança, Cuiabá, MT 78060-900, Brazil. E-mail: janessabreu@yahoo.com.br

This study evaluated the histomorphometric characteristics of tambaqui intestinal mucous after using Bacillus spp based probiotics, incorporated in feed and dissolved in water during transport. It was used a completely randomized design, with three treatments and seven replications, and a control treatment (fish fed commercial feed without probiotic) was compared with two other treatments involving the use of commercial probiotic incorporated in the feed or in the transport water. A total of 510 juvenile tambaqui (average weight and total length $83.26 \pm 28.14 \mathrm{~g}$ and $17.39 \pm 1.90 \mathrm{~cm}$, respectively) was distributed in three cement tanks. During 60 days, fish of two tanks were fed with commercial feed and fish of the third tank received commercial feed supplemented with probiotic. After this period, the fish from each tank were divided into plastic bags and transported for 4 hours according to the following treatments: $\mathrm{T} 1$ = feeding with commercial feed (control); $\mathrm{T} 2$ = feeding with commercial feed and probiotic dissolved in the water during transport $(20 \mathrm{mg} / \mathrm{L}) ; \mathrm{T} 3=$ feeding with commercial feed supplemented with probiotic $(1.0 \mathrm{~g} / \mathrm{kg}$ feed). Before transport (basaline), 24 and 96 hours after transport, eight fish of each treatment were submitted to euthanasia and the intestine was removed for weighing and length measuring to establish the relation between length/gut ratio. Then, the anterior and posterior portions of the intestine were collected for evaluation of morphological and histological characteristics of intestinal mucous. The use of probiotic during transport did not affect the intestine weight of tambaqui. Regardless of the treatment, the intestine length increased as the fish increased in size. The supplementation with probiotics (Bacillus spp.) had no effect on height and length villus of tambaqui's intestine during transport challenge.
\end{abstract}

INDEX TERMS: Bacillus spp., Colossoma macropomum, intestinal villous.

RESUMO.- Avaliou-se neste trabalho as características histomorfométricas da mucosa intestinal de tambaqui após uso de probiótico a base de Bacillus spp., veiculado

\footnotetext{
${ }^{1}$ Recebido em 18 de feverero de 2014.

Aceito para publicação em 3 de novembro de 2014.

${ }^{2}$ Programa de Pós Graduação em Ciência Animal, Universidade Federal de Mato Grosso (UFMT), Av. Fernando Corrêa da Costa 2367, Boa Esperança, Cuiabá, MT 78060-900, Brasil. E-mail: celmazootecnista@hotmail.com

${ }^{3}$ Faculdade de Agronomia, Medicina Veterinária e Zootecnia, UFMT, Av. Fernando Corrêa da Costa 2367, Boa Esperança, Cuiabá, MT 78060-900. E-mails: naassi@gmail.com, felipe.melhoramento@gmail.com, alexandra_
}

na ração e dissolvido na água durante transporte. Foi utilizado um delineamento inteiramente casualizado com três tratamentos e sete repetições, sendo comparado um

\footnotetext{
potenza@hotmail.com, thayssach.moraes@gmail.com; *Autor para correspondência: janessabreu@yahoo.com.br

${ }^{4}$ Faculdade de Medicina Veterinária e Zootecnia, Universidade Federal de Mato Grosso do Sul (UFMS), Av. Senador Filinto Müller 2443, Vila Ipiranga, Campo Grande, MS 79074-460, Brasil. E-mail: jayme.peixegen@ gmail.com

${ }^{5}$ Instituto de Biociências, UFMT, Av. Fernando Corrêa da Costa 2367, Boa Esperança, Cuiabá, MT 78060-900. E-mail: thaisteixeiraa2@hotmail.com
} 
tratamento controle (peixes alimentados com ração comercial sem probiótico) a dois outros tratamentos envolvendo o uso de probiótico comercial, veiculado na ração ou na água de transporte. Um total de 510 juvenis de tambaqui (peso e comprimento total médio inicial de $83,26 \pm 28,14 \mathrm{~g}$ e $17,39 \pm 1,90 \mathrm{~cm}$, respectivamente) foi distribuído em três tanques de alvenaria. Durante 60 dias, os peixes de dois tanques foram alimentados com ração comercial e os do terceiro tanque com ração comercial suplementada com probiótico. Após este período, os peixes de cada tanque foram divididos em sacos plásticos e transportados por 4 horas de acordo com os seguintes tratamentos: T1 = alimentação com ração comercial (controle); T2 = alimentação com ração comercial e probiótico adicionado na água durante o transporte $(20 \mathrm{mg} / \mathrm{L}) ; \mathrm{T} 3$ = alimentação com ração comercial suplementada com probiótico $(1,0 \mathrm{~g} / \mathrm{kg}$ de ração). Antes do transporte (basal), 24 e 96 horas após o transporte, oito peixes de cada tratamento foram submetidos a eutanásia e o intestino retirado para pesagem e mensuração do comprimento para estabelecimento da relação comprimento corporal/ intestino. Em seguida, foi realizada coleta da porção anterior e posterior do intestino, para avaliação das características morfo-histológicas da mucosa intestinal. 0 uso de probiótico durante o transporte não afetou o peso do intestino de juvenis de tambaqui. O comprimento do intestino do tambaqui não foi alterado pelo tratamento, sendo observada somente relação linear entre o comprimento intestinal e o comprimento corporal (CC) dos peixes. A suplementação com probiótico (Bacillus spp.) não exerceu nenhum efeito na altura e comprimento das vilosidades do intestino do tambaqui desafiados com o transporte.

TERMOS DE INDEXAÇÃO: Bacillus spp., Colossoma macropomum, vilosidades intestinais.

\section{INTRODUÇÃO}

Com a grande expansão do setor aquícola, a produção total da aquicultura no Brasil aumentou para 628.704 toneladas em 2011, representando um incremento de 31,1\% em relação à produção de 2010 (Brasil 2013). Segundo dados da FAO (2008), para o ano 2025 é esperado que a produção de pescado seja de 162 milhões de toneladas, baseando-se no consumo de $25 \mathrm{~kg}$ per capita/ano.

A criação em cativeiro do tambaqui (Colossoma macropomum) encontra-se, atualmente, em crescimento no Brasil, concentrada nas regiões Norte, Nordeste e Centro-Oeste (Lopera-Barrero et al. 2011). Trata-se de uma espécie onívora, cuja produção, juntamente com a da tilápia do Nilo (Oreochromis niloticus), representa 67,0\% da produção nacional de pescado (Brasil 2011).

Atualmente esta espécie é a segunda mais produzida no país, com mais de 111.084,1 toneladas (Brasil 2013). Grande parte do aumento da produção desta espécie, é pela facilidade de adaptação a alimentação artificial, rusticidade e resistência aos sistemas de cultivos, boa conversão alimentar e ótimo ganho de peso, sendo sua carne muito apreciada e com alto valor nutritivo (Gomes et al. 2010, Lopera-Barrero et al. 2011).
Com esse aprimoramento da atividade aquícola, juntamente com o aumento da criação intensiva de peixes, há uma preocupação por parte dos criadores que a intensificação dos cultivos possa causar estresse. Entre os vários malefícios que o estresse pode causar à saúde dos peixes, está a redução da competência imunológica, deixando-os mais vulneráveis às infecções bacterianas e parasitoses (Garcia 2008). Quando peixes são expostos à estímulos desagradáveis, como os que ocorrem durante os procedimentos de transporte, uma das respostas fisiológicas apresentadas é a perda da capacidade imunológica (Urbinati \& Carneiro 2004) e nesta condição de maior susceptibilidade, bactérias com potencial patogênico que compõem a microbiota intestinal dos peixes podem provocar doenças

Uma alternativa que atualmente vem sendo incorporada às pisciculturas e que tem trazido benefícios ao sistema imune dos peixes é o uso de probióticos, microrganismos vivos que quando adicionados adequadamente às dietas promovem benefícios ao hospedeiro (Verschuere et al. 2000). As bactérias probióticas entram no trato digestivo diretamente pela água ou através de ração inoculada e podem ser usadas na aquicultura como condicionadoras de qualidade da água e como suplemento alimentar. No trato gastrointestinal, o probiótico pode provocar alteração da microbiota intestinal, modulação da resposta imune e ajuda nas funções metabólicas, proporcionando um melhor crescimento, bom estado de saúde e resistência à doenças (Kiron 2012).

Neste sentido, probióticos podem ser utilizados com a função de reduzir a carga das bactérias indesejáveis do trato intestinal dos peixes, favorecendo a saúde do animal e melhorando a absorção dos nutrientes da dieta. Os probióticos mais estudados em peixes são os compostos por $\mathrm{Ba}$ cillus subtilis, que apresenta propriedades benéficas quando suplementado às dietas, incluindo imunoestimulação e resistência à doenças (Cerezuela et al. 2012).

0 intestino é um órgão envolvido em importantes funções fisiológicas, sendo o principal local de digestão dos alimentos e absorção de nutrientes (Caballero et al. 2003). A mucosa intestinal é fundamental nos processos digestivos, absortivo e metabólico em peixes teleósteos e o aumento do comprimento das vilosidades implica em um aumento da área de superfície para maior absorção dos nutrientes disponíveis (Caspary 1992). Pesquisas com probióticos para animais aquáticos têm avaliado os efeitos positivos sobre a morfologia intestinal dos animais, como benefícios na estrutura de microvilosidades e superfície absortiva (Rahiman et al. 2010, Carvalho et al. 2011, Mello et al. 2013).

0 transporte de peixes quando mal planejado e executado pode levar a grandes prejuízos ao produtor, face à mortalidade frequentemente observada (Urbinati \& Carneiro 2004). 0 objetivo com este estudo foi avaliar as características histomorfométricas da mucosa intestinal de tambaqui (C. macropomum) após uso de probiótico a base de Bacillus spp., veiculado na ração e dissolvido na água durante transporte.

\section{MATERIAL E MÉTODOS}

O experimento foi conduzido na Estação Experimental de Piscicultura da Fazenda Experimental da Faculdade de Agronomia, Medicina Veterinária e Zootecnia (FAMEVZ) da Universidade Federal de Mato 
Grosso (UFMT) e foi aprovado pelo Comitê de ética em Uso de Animais (CEUA/UFMT) sob o número de protocolo 23108.058693/130 , seguindo os princípios éticos para pesquisa animal determinados pelo Colégio Brasileiro de Experimentação Animal (Cobea 1991).

\section{Protocolo experimental}

Um total de 510 juvenis de tambaquis $(83,26 \pm 28,14 \mathrm{~g}$ e $17,39 \pm 1,90 \mathrm{~cm}$ ), capturado em viveiro escavado foi estocado dentro do laboratório em três tanques de alvenaria de 1000 litros cada, na densidade de 170 peixes/tanque, abastecidos com fluxo de água e aeração constantes. Após aclimatação, que foi considerada terminada quando todos os peixes estavam se alimentando normalmente, teve início o período experimental de 60 dias, no qual os peixes de dois tanques foram alimentados com ração comercial extrusada para peixes onívoros cuja composição bromatológica é expressa no Quadro 1, enquanto os do terceiro tanque foram alimentados com a mesma ração comercial acrescida de probiótico $(1,0 \mathrm{~g}$ de probiótico/kg de ração). 0 probiótico foi primeiramente dissolvido em água $(100 \mathrm{~mL})$ e incorporado na ração comercial através de borrifação, seguindo as recomendações do fabricante. A alimentação foi realizada duas vezes ao dia (manhã e tarde) até saciedade aparente.

Após este período, os peixes estocados nos tanques de alvenaria foram privados de alimentação por 24 horas, para esvaziamento do trato gastrointestinal. Em seguida, 70 peixes foram retirados aleatoriamente de cada tanque, distribuídos em sete sacos abastecidos com 15 litros de água (10 peixes/saco; 7 sacos /tratamento) e submetidos ao transporte de acordo com os seguintes tratamentos T1:alimentação com ração comercial (controle); T2: alimentação com ração comercial e probiótico dissolvido na água durante o transporte $(20 \mathrm{mg} / \mathrm{L})$; T3: alimentação com ração comercial suplementada com probiótico $(1,0 \mathrm{~g} / \mathrm{kg}$ de ração). Após serem amarrados, os sacos foram colocados em carroceria de um

\section{Quadro 1. Características e composição nutricional da ração} comercial utilizada

\begin{tabular}{|c|c|c|}
\hline Tipo & Item & Quantidade \\
\hline Características & Umidade (máx.) & $110 \mathrm{~g} / \mathrm{kg}$ \\
\hline \multirow[t]{4}{*}{ Macronutrientes } & Proteína Bruta (min.) & $320 \mathrm{~g} / \mathrm{kg}$ \\
\hline & Extrato Etéreo (min.) & $50 \mathrm{~g} / \mathrm{kg}$ \\
\hline & Matéria Mineral (máx.) & $110 \mathrm{~g} / \mathrm{kg}$ \\
\hline & Matéria Fibrosa (máx.) & $70 \mathrm{~g} / \mathrm{kg}$ \\
\hline Macrominerais e & Cálcio (min.) & $20 \mathrm{~g} / \mathrm{kg}$ \\
\hline \multirow[t]{9}{*}{ Microminerais } & Cálcio (máx.) & $30 \mathrm{~g} / \mathrm{kg}$ \\
\hline & Fósforo (min.) & $15 \mathrm{~g} / \mathrm{kg}$ \\
\hline & Sódio (min.) & $370 \mathrm{mg} / \mathrm{kg}$ \\
\hline & Cobalto (min.) & $0,75 \mathrm{mg} / \mathrm{kg}$ \\
\hline & Ferro (min.) & $75 \mathrm{mg} / \mathrm{kg}$ \\
\hline & Iodo (min.) & $1,5 \mathrm{mg} / \mathrm{kg}$ \\
\hline & Manganês (min.) & $7,5 \mathrm{mg} / \mathrm{kg}$ \\
\hline & Cobre (min.) & $4,5 \mathrm{mg} / \mathrm{kg}$ \\
\hline & Selênio (min.) & $0,3 \mathrm{mg} / \mathrm{kg}$ \\
\hline \multirow[t]{14}{*}{ Vitaminas } & Ácido Fólico (min) & $5,1 \mathrm{mg} / \mathrm{kg}$ \\
\hline & Ácido Pantotênico (min.) & $17 \mathrm{mg} / \mathrm{kg}$ \\
\hline & Biotina (min.) & $0,85 \mathrm{mg} / \mathrm{kg}$ \\
\hline & Vitamina A (min.) & 6800 U.I/kg \\
\hline & Vitamina B1 (min.) & 17 mg/kg \\
\hline & Vitamina B12 (min.) & $25,5 \mathrm{mcg} / \mathrm{kg}$ \\
\hline & Vitamina B2 (min.) & $17 \mathrm{mg} / \mathrm{kg}$ \\
\hline & Vitamina B6 (min.) & 17 mg/kg \\
\hline & Vitamina C (min.) & $300 \mathrm{mg} / \mathrm{kg}$ \\
\hline & Vitamina D (min.) & $2550 \mathrm{U.I} / \mathrm{kg}$ \\
\hline & Vitamina E (min.) & 102 U.I/kg \\
\hline & Vitamina K3 (min) & $3,4 \mathrm{mg} / \mathrm{kg}$ \\
\hline & Niacina (min.) & $34 \mathrm{mg} / \mathrm{kg}$ \\
\hline & Colina (min.) & $850 \mathrm{mg} / \mathrm{kg}$ \\
\hline
\end{tabular}

veículo e os peixes transportados por 4 horas, o que representa o transporte médio de curta distância no Estado de Mato Grosso.

Parâmetros de qualidade de água, como temperatura da água $\left(28,45 \pm 0,17^{\circ} \mathrm{C}\right)$, oxigênio dissolvido $(2,00 \pm 0,008 \mathrm{mg} / \mathrm{L})$ (oxímetro digital Yellow Springs Instruments (YSI) 55), $\mathrm{pH}(7,00 \pm 0,02)$ (peagâmetro digital Quimis ${ }^{\circledR}$ Q400BC), alcalinidade $=205,60 \pm 3,89$ mg de $\mathrm{CaCO}_{3} / \mathrm{L}$ (a partir da solução indicadora de metil laranja) e amônia não ionizada $(0,0214 \pm 0,0051 \mathrm{mg} / \mathrm{L}$ ) (calculada segundo Emerson et al. 1975) foram monitorados durante o período experimental. 0 sifonamento do fundo dos tanques de alvenaria e a renovação de água impediram o acúmulo de matéria orgânica.

\section{Análise biométrica do intestino}

Antes do transporte (basal), 24 e 96 horas após este procedimento, oito peixes de cada tratamento, foram anestesiados com solução de eugenol $(20 \mathrm{ml}$ de óleo de cravo diluído em álcool etílico $92,8^{\circ}$, seguido da diluição de $30 \mathrm{ml}$ desta solução estoque em 15 litros de água), pesados (balança de precisão AS 2000 C- Marte) e submetidos à eutanásia através de secção encefálica. Em seguida, foi realizada uma incisão na cavidade abdominal dos peixes para retirada do intestino, o qual foi separado do estômago, para mensuração do peso (balança de precisão AS 2000 C- Marte) e comprimento (ictiômetro), utilizados no estabelecimento do peso relativo (peso do intestino/ peso do peixe x 100) e da relação comprimento do intestino/ comprimentocorporal (CI/CC).

\section{Análise morfohistológicadas vilosidades intestinais}

Após retirada do intestino, foi realizada coleta de fragmento $(2 \mathrm{~cm})$ da porção anterior e posterior, as quais foram fixadas em formalina $10 \%$ por 24 horas e conservadas em álcool $70 \%$ para avaliação das características morfo-histológicas da mucosa intestinal (altura e comprimento das vilosidades). 0 processamento histológico das amostras ocorreu no Laboratório de Patologia Veterinária da Universidade Federal de Mato Grosso (UFMT) conforme processamento rotineiro para preparação de lâminas histológicas que foram coradas com hematoxilina e eosina (HE), seguindo a metodologia de Prophet et al.(1992).

Em cada lâmina confeccionada, foram medidos a altura e comprimento, em micra $(\mu \mathrm{m})$, de oito vilosidades, tanto do fragmento da porção anterior (Fig.1A) como da posterior (Fig.1B). A altura foi medida do ápice à base de cada uma das oito vilosidades (Fig. $2 \mathrm{~A}$ ), e o comprimento foi obtido com as medidas do perímetro de cada uma das vilosidades (Fig.2B) através da digitalização das amostras utilizando o Software analisador de imagens Axio.

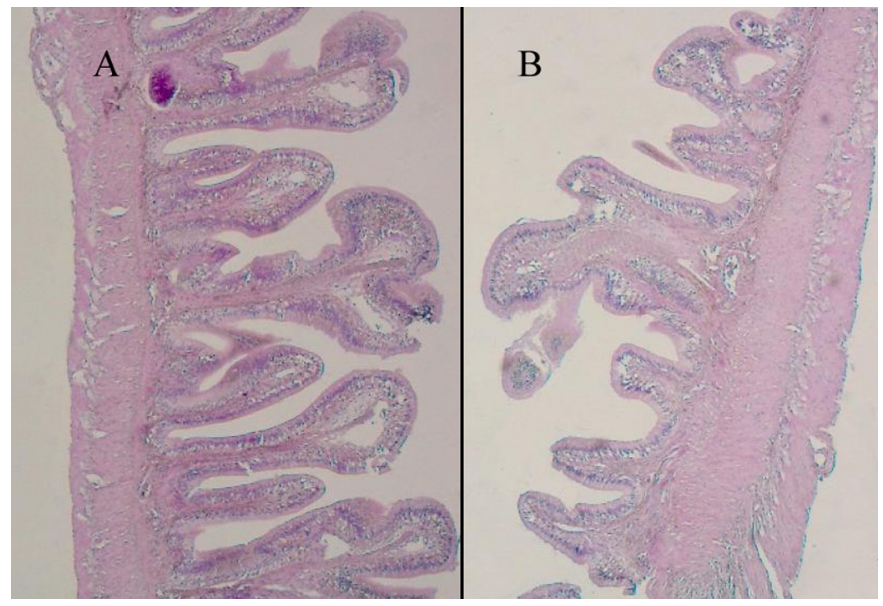

Fig.1. Vilosidades do intestino de tambaqui (Colossoma macropomum) na porção anterior (A) e posterior (B) do intestino. HE, obj.5x. 


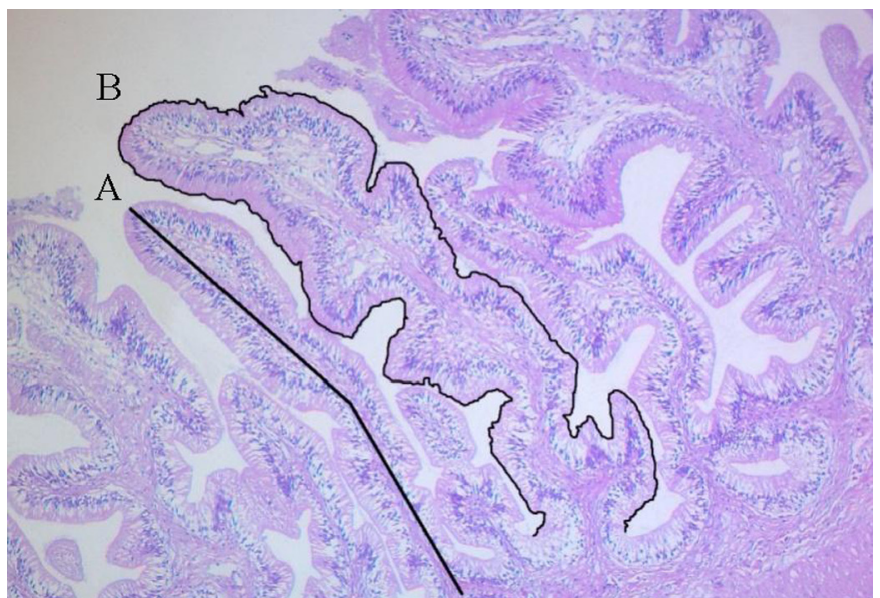

Fig.2. Vilosidades do intestino de tambaqui (Colossoma macropomum). A) Altura das vilosidades; B) Comprimento das vilosidades. HE, obj.5x.

\section{Análises estatísticas}

0 experimento foi delineado de forma inteiramente casualizada, com três classes para o fator avaliado: sem adição de probiótico (T1), adição de probiótico na água de transporte (T2) e adição de probiótico na ração (T3). As variáveis respostas avaliadas foram: altura e comprimento das vilosidadesintestinais anteriores e posteriores $(\mu \mathrm{m})$ e peso relativo (\%). As medidas foram tomadas e analisadas nos tempos basal, 24 horas e 96 horas. As análises realizadas para dados coletados no tempo basal foram realizadas por meio de contrastes, pois os tratamentos T1 e T2 não continham diferenças uma vez que os peixes destes tratamentos não tinham recebido o probiótico durante os 60 dias de experimento. 0 modelo estatístico utilizado na análise foi apresentado na Fórmula 1:

$$
y_{i j}=\mu+t_{i}+\beta_{i j}
$$

em que:

$\mathrm{y}_{i j} \mathrm{y}_{i j}=$ Valor médio observado em cada peixe para as características avaliadas;

$\mu \mu=$ Efeito fixo da média geral do experimento;

$\mathrm{t}_{\mathrm{t}}=$ Efeito fixo do tratamento $i$;

$\theta_{i j} \theta_{i j}=$ Efeito aleatório residual incidente sobre a média de cada peixe; $i i=$ sub-índice que indica o tratamento incidente;

$j j=$ sub-índice que indica o peixe analisado.

As análises estatísticas foram feitas utilizando o software SAS (versão 9.0), sendo os dados do Modelo 1 submetidos à Análise de Variância (utilizando mínimos quadrados ponderados) e a comparação entre médias realizada através do Teste de Tukey ao nível de $5 \%$ de probabilidade.

Além destas análises, foi realizado um estudo observacional entre as características comprimento corporal dos peixes e comprimento do intestino, através de uma análise de variância da regressão, considerando as variações com comprimento total como função das variações do comprimento do intestino. 0 modelo estatístico utilizado, testando apenas o efeito linear, pode ser encontrado na Fórmula 2.

$$
\mathrm{y}_{i=} \beta_{0+} \beta_{1} x_{i+} \theta_{i}
$$

em que:
$y_{i} y_{i}=$ observação do comprimento peixe $i$;
$\beta_{\eta} \beta_{\eta}=$ Intercepto da equação;
$\beta_{1} \beta_{1}=$ Coeficiente da regressão linear;
$x_{i} X_{i}=$ Medida do comprimento do intestino do peixe $i$;
$\theta_{i} \theta_{i}=$ Efeito aleatório residual incidente em cada observação.

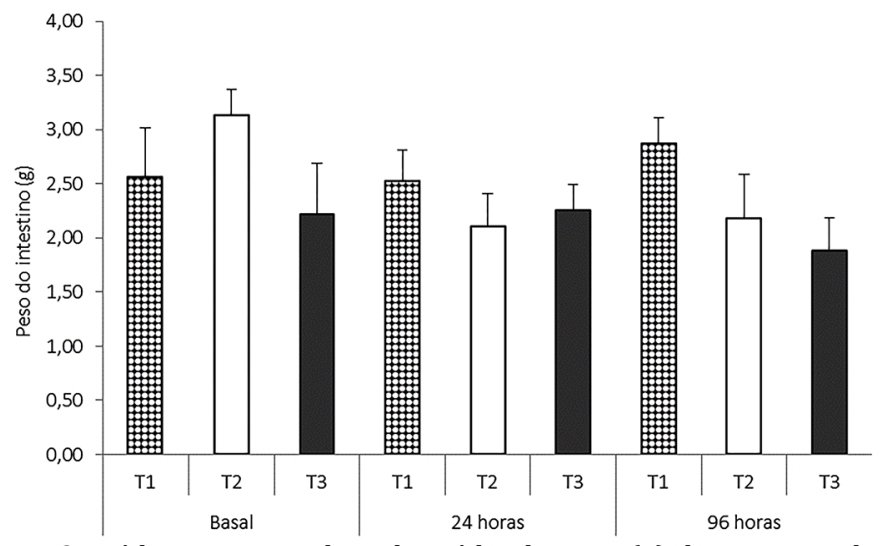

Fig.3. Médias \pm erro padrão da média do peso ( $g$ ) do intestino de juvenis de tambaqui (Colossoma macropomum) submetidos ao transporte com uso de probiótico. $\mathrm{T} 1$ = Sem probiótico na ração comercial e na água do transporte (controle). T2 = Alimentação com ração comercial e probiótico adicionado na água do transporte $(20 \mathrm{mg} / \mathrm{L})$. T3 = Alimentação com ração comercial suplementada com probiótico $(1,0 \mathrm{~g} / \mathrm{kg}$ de ração).

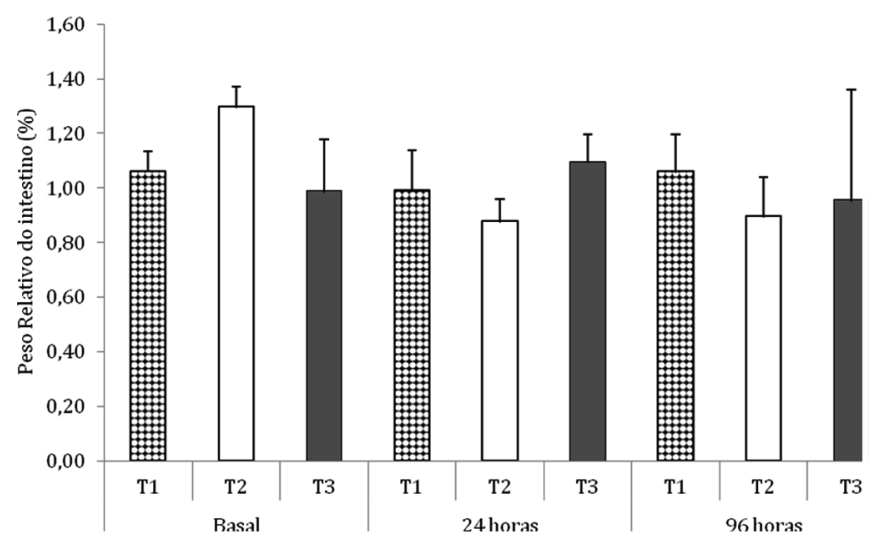

Fig.4. Médias \pm erro padrão da média do peso relativo (\%) do intestino de juvenis de tambaqui (Colossoma macropomum) submetidos ao transporte com uso de probiótico. T1 = Sem probiótico na ração comercial e na água do transporte (controle). T2 = Alimentação com ração comercial e probiótico adicionado na água do transporte $(20 \mathrm{mg} / \mathrm{L})$. T3 = Alimentação com ração comercial suplementada com probiótico $(1,0 \mathrm{~g} / \mathrm{kg}$ de ração).

\section{RESULTADOS}

Não foi registrada mortalidade durante o período experimental. Os resultados da avaliação biométrica mostraram que o uso de probiótico durante o transporte não exerceu efeito significativo $(\mathrm{P}>0,05)$ no peso (g) (Fig.3) e peso relativo (\%) (Fig.4) do intestino de juvenis de tambaqui. Da mesma forma, o comprimento do intestino do tambaqui não foi alterado pelo uso de probiótico, sendo observada somente relação linear entre o comprimento intestinal (CI) e o comprimento corporal (CC) dos peixes ( $\mathrm{P}<0,0001$; $r^{2}=0,301$ ) (Fig.5).

A análise morfo-histológica do intestino mostrou que a altura e comprimento das vilosidades da mucosa intestinal não diferiram significativamente $(P>0,05)$ entre os peixes transportados com probiótico ou alimentados por 60 dias com ração contendo o aditivo quando comparado aos peixes do tratamento controle (Quadro 2). 


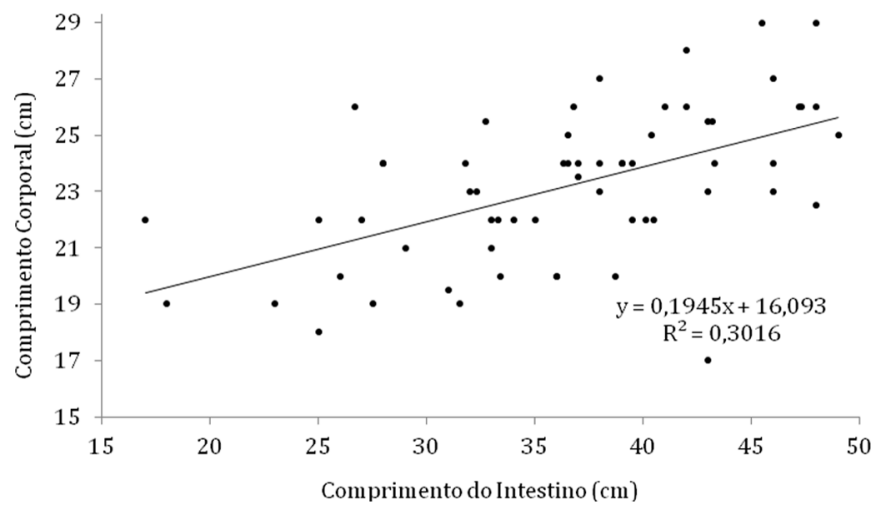

Fig.5. Relação entre o comprimento corporal e comprimento do intestino de juvenis de tambaqui (Colossoma macropomum) submetidos ao transporte com uso de probiótico.

\section{DISCUSSÃO}

Os parâmetros físico-químicos da água, avaliados durante o período experimental, apresentaram valores médios dentro do adequado à criação de peixes, exceto pelos níveis de oxigênio dissolvido, cujos valores concentraram-se em torno de 2,0mg/L, abaixo do recomendado para espécies tropicais (Boyd \& Tucker 1998). Contudo, estes baixos valores de oxigênio não foram aparentemente prejudiciais aos tambaquis estudados neste trabalho, uma vez que mortalidade não foi registrada durante todo o período experimental. Por ser uma espécie autóctone da Bacia amazônica, o tambaqui é adaptado às variações extremas de oxigênio que ocorrem em determinados períodos do ano em seu habitat natural, o que o torna resistente a baixos níveis de oxigênio dissolvido quando criado em cativeiro.

É de conhecimento científico que probióticos interferem nas características anatômicas do trato gastrointestinal, pois promovem o aumento na superfície de absorção da mucosa intestinal, proporcionando incremento do comprimento intestinal e melhorando o desempenho (Silva \& Nörnberg 2003).
Alterações no comprimento do intestino podem ser compensadas por variações na área da mucosa intestinal e pela variabilidade de tipos celulares encontrada no epitélio dessa mucosa (Borges et al. 2010). Em larvas de tilápias-do-Nilo foi observado aumento linear sobre o comprimento do intestino com aumento nos níveis de prebiótico a base de mananoligossacarídeo (MOS) nas dietas (Schwarz et al. 2011). No presente trabalho, o peso e comprimento do intestino de tambaqui não foram significativamente diferentes frente ao uso de probiótico na ração ou dissolvido na água durante o transporte. Resultados semelhantes foram obtidos em outros animais de produção, como os relatados por Otutumi (2006) que verificou que não houve influência do probiótico $\left(1,6 \times 10^{9} \mathrm{UFC} / \mathrm{g}\right.$ de Lactobacillus spp.) no comprimento do intestino delgado de codornas de corte (Coturnix coturnix sp.). Além disso, correlação linear positiva foi verificada na relação crescimento corporal/ crescimento do intestino do tambaqui, sendo que o crescimento do intestino acompanhou proporcionalmente o comprimento-padrão dos peixes.

Há relatos na literatura de que a relação comprimento do intestino/comprimento corporal (CI/CC) varia de acordo com hábito alimentar, sendo de 0,2 a 2,5 nos peixes carnívoros; entre 0,6 e 8,0 nos onívoros e de 0,8 a 15,0 e nos herbívoros (Rotta 2003). A alimentação natural do tambaqui, espécie estudada neste trabalho, inclui zooplâncton, frutos e sementes, sendo classificada como uma espécie onívora de fácil aceitação às rações artificiais e adaptação à criação em cativeiro (Lopera-Barrero et al. 2011). Independente do uso do probiótico, neste estudo, o comprimento médio do intestino de tambaqui foi de $36,36 \pm 2,99 \mathrm{~cm}$ e a relação CI/CC média obtida foi de 1,56 $\pm 0,04$ corroborando com o relatado na literatura para peixes onívoros (Rust 2002, Baldisserotto 2009).

A integridade da mucosa intestinal pode ser avaliada a partir de mensurações da altura e densidade dos vilos. 0 equilíbrio entre renovação celular e perda de células que ocorre normalmente no intestino determina uma reno-

\begin{abstract}
Quadro 2. Médias \pm erro padrão da média da altura e comprimento de vilosidades da porção anterior e posterior do intestino de juvenis de tambaqui (Colossoma macropomum) submetidos ao transporte com uso de probiótico. T1=Sem probiótico na ração comercial e na água do transporte (controle). T2=Alimentação com ração comercial e probiótico adicionado na água do transporte $(20 \mathrm{mg} / \mathrm{L})$. T3=Alimentação com ração comercial suplementada com probiótico $(1,0 \mathrm{~g} / \mathrm{kg}$ de ração)
\end{abstract}

\begin{tabular}{|c|c|c|c|c|c|}
\hline \multirow[t]{3}{*}{ Tempos } & & \multicolumn{4}{|c|}{ Parâmetros } \\
\hline & & \multicolumn{2}{|c|}{ Altura das vilosidades } & \multicolumn{2}{|c|}{ Comprimento das vilosidades } \\
\hline & & Anterior $(\mu \mathrm{m})$ & Posterior $(\mu \mathrm{m})$ & Anterior $(\mu \mathrm{m})$ & Posterior $(\mu \mathrm{m})$ \\
\hline \multirow[t]{3}{*}{ Basal } & $\mathrm{T} 1$ & $324,51 \pm 38,29^{a}$ & $334,15 \pm 61,97^{\mathrm{a}}$ & 1010,77 & $974,65 \pm 199,40^{\mathrm{a}}$ \\
\hline & $\mathrm{T} 2$ & $463,41 \pm 92,78^{a}$ & $363,65 \pm 100,95^{a}$ & 1330,7 & $326,07^{\mathrm{a}}$ \\
\hline & $\mathrm{T} 3$ & $368,40 \pm 21,08^{a}$ & $311,19 \pm 25,37^{a}$ & $1017,01 \pm 45,35^{\mathrm{a}}$ & $994,67 \pm 120,56^{a}$ \\
\hline \multirow[t]{3}{*}{24 horas } & $\mathrm{T} 1$ & $420,81 \pm 88,22^{a}$ & $281,93 \pm 56,30^{\mathrm{a}}$ & $1167,4 \pm$ & $733,03 \pm 111,74^{\mathrm{a}}$ \\
\hline & $\mathrm{T} 2$ & $391,40 \pm 70,97^{\mathrm{a}}$ & $475,54 \pm 147,20^{a}$ & 1269, & $178,30^{\mathrm{a}}$ \\
\hline & $\mathrm{T} 3$ & $332,15 \pm 47,41^{\mathrm{a}}$ & $303,90 \pm 46,04^{a}$ & $892,0 \pm 110,15^{\mathrm{a}}$ & $865,6 \pm 112,71^{a}$ \\
\hline \multirow[t]{3}{*}{96 horas } & $\mathrm{T} 1$ & $287,59 \pm 18,76^{a}$ & $246,92 \pm 39,59^{a}$ & $786,2 \pm 81,89^{a}$ & $708,5 \pm 124,20^{\mathrm{a}}$ \\
\hline & $\mathrm{T} 2$ & $280,89 \pm 49,86^{a}$ & $228,01 \pm 38,13^{a}$ & $723,3 \pm 122,03^{\mathrm{a}}$ & $673,0 \pm 133,49^{a}$ \\
\hline & T3 & $231,38 \pm 28,92^{a}$ & $247,12 \pm 61,35^{a}$ & $577,3 \pm 40,82^{\mathrm{a}}$ & $749,5 \pm 266,29^{a}$ \\
\hline
\end{tabular}

Letras minúsculas diferentes na coluna indicam diferenças significativas nos parâmetros avaliados entre tratamentos. 
vação celular constante, mantendo o tamanho dos vilos e por consequência a capacidade digestiva e de absorção intestinal. Contudo, quando algum agente, como microrganismos, entram em contato com o intestino, pode ocorrer um desequilíbrio e interferência nesta renovação celular, modificando a altura, comprimento e espessura das vilosidades (Pelicano et al. 2003). Estudos mostram que larvas, alevinos e juvenis de tilápias do Nilo (Oreochromis niloticus) apresentaram maior altura de vilosidade intestinal e espessura do epitélio da mucosa quando alimentados com dietas suplementadas com probióticos, a base de Bacillus cereus e Bacillus subtilis, ou prebióticos a base de mananoligossacarídeo (MOS) (Carvalho et al. 2011, Schwarz et al. 2011, Cechim 2012, Mello et al. 2013). Em outras espécies, como larvas de bijupirá (Rachycentron canadum) (Salze et al. 2008) e juvenis de truta arco íris (Oncorhynchus mykiss) (Yilmaz et al. 2007) foi encontrado maior altura nas vilosidades intestinais após suplementação com MOS na dieta.

Apesar da literatura relatar efeitos benéficos do uso destes aditivos na mucosa intestinal em algumas espécies de peixes (Hisano et al. 2006, Carvalho et al. 2011, Schwarz et al. 2011, Mello et al. 2013), pela análise morfológica realizada neste estudo, pode-se considerar que não houve alterações nas características morfológicas do trato intestinal, indicando que, independente da forma como foi aplicado, o probiótico utilizado não exerceu efeito sobre o aumento da área de absorção da mucosa intestinal do tambaqui. Semelhante a este trabalho, em estudo sobre a caracterização histomorfométrica de intestino de surubins (Pseudoplatystoma ssp.) alimentados por 20 dias com dieta suplementada com probiótico (Bacillus subtilis) nas doses de 10, 20, 30 e 40g/kg de ração,a análise de regressão indicou efeito linear negativo dos níveis de probiótico sobre a altura dos vilos intestinais,sugerindo que não houve benefício do aditivo na histomorfometria intestinal (Nunes 2013).

Resultados com pesquisas envolvendo o uso de probióticos são bastante contraditórios quanto à sua eficiência e podem variar em relação à espécie de animal estudada, idade do animal, tipo de probiótico utilizado, viabilidade de microrganismos a serem incorporados às rações e suas condições de armazenamento (Araujo et al. 2007). Faria et al. (2009), afirmam que vários aspectos como dosagem inadequada dos microrganismos que compõem os probióticos, falta de desafio sanitário em condições experimentais e uma possível competição com o hospedeiro por nutrientes podem contribuir para a não expressão de respostas favoráveis quando da utilização de probióticos.

Diferente das pesquisas com probióticos realizadas em outras espécies de peixes em que a suplementação envolvia uma cepa específica de microrganismo, ou seja, apenas Bacillus subtilis ou Bacillus cereus, o probiótico comercial utilizado neste estudo era composto por uma mistura de Bacillus (B. licheniformis, B. subtilis e B. pumilus). Espécies como B. subtilis e B. licheniformis ocorrem naturalmente no trato digestório de camarões, de forma que o produto comercial testado neste trabalho é utilizado amplamente na carcinicultura, com efeitos benéficos reconhecidos (Decamp et al. 2008). Assim, para que um probiótico seja considerado efetivo, ele deve apresentar algumas proprie- dades básicas, como a de permanecer no ecossistema intestinal do hospedeiro e ser isolado da mesma espécie animal a que se destina (Saarela et al. 2000). Portanto, a relação probiótico hospedeiro parece ser espécie-específica e pode ser que as cepas de Bacillus ou a densidade delas presente no produto comercial utilizado neste trabalho não sejam adequadas para serem utilizadas como probiótico em tambaqui. Neste caso, o isolamento e a seleção de bactérias benéficas específicas do trato intestinal de tambaquis constituem-se importante estratégia para o desenvolvimento de probióticos para essa espécie.

Outro fator que limita o uso de probióticos, em muitos casos, é a não manutenção da concentração dos mesmos no trato intestinal dos animais, existindo assim a necessidade de que sejam oferecidos regularmente aos organismos aquáticos, em altas concentrações, para se obter o efeito desejado. Neste estudo, as concentrações testadas não variaram $(1,0 \mathrm{~g} / \mathrm{kg}$ na dieta e $20 \mathrm{mg} / \mathrm{L}$ na água de transporte) e eram menores do que as concentrações testadas em trabalhos com outras espécies de peixes.

O conhecimento básico da fisiologia digestiva dos peixes é fundamental para o desenvolvimento de dietas que atendam as exigências nutricionais das espécies cultivadas (Gonçalves et al. 2012). Contudo, são escassas as informações disponíveis na literatura envolvendo a morfofisiologia do sistema digestivo de peixes, especialmente em espécies nativas. Nas condições em que foi realizado este experimento, a inclusão de probiótico comercial a base de Bacillus spp na ração $(1,0 \mathrm{~g} /$ $\mathrm{kg}$ ) ou na água (20mg/L) não influenciou o peso do intestino, altura e comprimento das vilosidades intestinais de juvenis de tambaqui (C. macropomum) desafiados com o estresse de transporte. Pesquisas adicionais envolvendo diferentes tipos de probióticos e maiores concentrações são necessárias para se entender o efeito destes aditivos na mucosa intestinal e desempenho zootécnico de tambaquis cultivados.

Agradecimentos.- À Coordenação de Aperfeiçoamento de Pessoal de Nível Superior (CAPES) pela concessão de bolsa de mestrado à autora Celma M. Ferreira. Ao Conselho Nacional de Desenvolvimento Científico e Tecnológico (CNPq) pela bolsa de produtividade em pesquisa concedida ao autor Jayme A. Povh. À Piscicultura São João pela doação dos peixes. À empresa Matsuda pela doação da ração utilizada no experimento.

\section{REFERÊNCIAS}

Araujo J.A., Silva J.H.V., Amâncio A.L.L., Lima M.R. \& Lima C.B. 2007. Uso de aditivos naalimentação de aves. Acta Vet. Brasilica 1:69-77.

Baldisserotto B. 2009. Fisiologia de peixes aplicada à piscicultura. $2^{\underline{a}}$ ed. Ed. da UFSM, Santa Maria, p.27-31.

Borges J.C.S., Sanches E.G., Oliveira M.S. \& Silva J.R.M.C. 2010. Anatomia e histologia gastrintestinal da garoupa- verdadeira Epinephelus marginatus (Lowe,1834) (Teleostei, Serranidae). Actascibiolsci., Maringá, 32(4):407-414.

Boyd C.E. \& Tucker C.S. 1998. Ecology of aquaculture ponds, p. 8-86. In: Ibid. (Eds), Pond Aquaculture Water Quality Management. Kluwer Academic Publishers, Boston, Massachusetts.

Brasil 2013. Boletim estatístico da pesca e aquicultura 2011. Ministério da pesca e aquicultura. Disponível em <http://www.mpa.gov.br> Acesso em 14 out. 2013.

Caballero M.J., Izquierdo M.S., Kjørsvik E., Socorro D.J.M., Fernandez A.J. \& RosenlundG. 2003. Morphological aspects of intestinal cells from gilthead seabream (Sparus aurata) fed diets containing different lipid sources. Aquaculture 225:325-340. 
Carvalho J.V., Lira A.D., Costa D.S.P., Moreira E.L.T., Pinto L.F.B. \& Albinati R.C.B. 2011. Desempenho zootécnico e morfometria intestinal de alevinos de tilápia-do-Nilo alimentados com Bacillus subtilisou mananoligossacarídeo. Revta Bras. Saúde Prod. Anim. 12:176-187.

Caspary W.F. 1992. Physiology and pathophysiology of intestinal absorption. Am. J. Clin. Nutr. 55:299-308.

Cechim F.E. 2012. Características morfológicas do epitélio intestinal e desempenho de Tilápia-do-Nilo, Oreochromis niloticus, suplementada com mananoligossacarídeo (MOS). Dissertação de Mestrado em Produção Animal, Programa de Pós-Graduação em Zootecnia, Universidade Tecnológica Federal do Paraná, Dois Vizinhos, Paraná.

Cerezuela R., Guardiola F.A., González P., Meseguer J. \& Esteban M.A. 2012. Effects of dietary Bacillus subtilis, Tetraselmischuii, and Phaeodactylum tricornutum, singularly or in combination, on the immune response and disease resistance of sea bream (Sparus aurata L.). Fish and Shellfish Immun. 33:342-349.

Cobea 1991. Princípios Éticos na Experimentação Animal. Colégio Brasileiro de Experimentação Animal. Disponível em <http://www.cobea. org. br/etica.htm\#3> Acesso em 26 out. 2013.

Decamp 0., Moriarty D.J.W. \& Lavens P. 2008. Probiotics for shrimp larviculture: review of field data from Asia and Latin America. Aquac. Res. 39:334-338.

Emerson K., Russo R.C., Lund R.E. \& Thurston R.V. 1975. Aqueous ammonia equilibrium calculations: Effects of $\mathrm{pH}$ and temperature. J. Fish. Res. Board Can. 32:2379-2383.

FAO. 2008. Fisheries Department, Fishery Information Data and Statistics Unit. Fish stat plus: universal software for fishery statistical time series. Aquaculture production: quantities 1950-2007, Aquaculture production: values 1984-2007; Capture production: 1950-2007. Version 2.30. Rome: FAO, 2008. Disponível em <http://www.fao.org> Acesso em 26 out. 2013

Faria D.E., Henrique A.P.F., Franzolin Neto R., Medeiros A.A., Junqueira O.M. \& Faria Filho D.E. 2009. Alternativas ao uso de antibióticos como promotores de Crescimento para frangos de corte: Probióticos. Ciênc. Anim. Bras. 10:18-28

Garcia F. 2008. Suplementação alimentar com $\beta$-glucano e mananoligossacarídeo para tilápias do Nilo em tanques-rede. Tese de Doutorado em Aquicultura, Centro de Aquicultura, Universidade Estadual Paulista, Jaboticabal, SP. 100p.

Gomes L.C., Simões L.N. \& Araújo-Lima C.A.R.M. 2010. Tambaqui (Colossoma macropomum). In: Baldisserotto B. \& Gomes L.C. (Eds), Espécies Nativas para Piscicultura no Brasil. UFSM, Santa Maria, RS.

Gonçalves L.U., Rodrigues A.P.O., Moro G.V., Cargnin-Ferreira E. \& Cyrino J.E.P. 2012. Morfologia e Fisiologia do Sistema Digestório de Peixes, p.9. In: Fracalossi D.M. \& Cyrino J.E.P. (Eds), NUTRIAQUA: nutrição e alimentação de espécies de interesse para a aquicultura brasileira. Sociedade Brasileira de Aquicultura e Biologia Aquática, Florianópolis, SC.

Hisano H., Silva M.D.P., Barros M.M. \& Pezzato L.E. 2006. Levedura íntegra e derivados de seu processo em rações para tilápia do Nilo: aspectos hematológicos e histológicos. Acta Sci. Biol. 28(4):311-8.

Kiron V. 2012. Fish immune system and its nutritional modulation for preventive health care. Anim. Feed Sci. Technol. 173:111-133.
Lopera-Barreto M.N., Ribeiro R.P., Povh J.A., Mendes L.D.V. \& Poveda-Parra A.R. 2011. Produção de organismos aquáticos: Uma visão geral do Brasil e no Mundo. Editora Agro livros, Guaíba, RS. 320p.

Mello H., Moraes J.R.E., Niza I.G., Moraes F.R., Ozório R.O.A., Shimada M.T., Engracia Filho J.R. \& Claudiano G.S. 2013. Efeitos benéficos de probióticos no intestino de juvenis de Tilápia-do-Nilo. Pesq. Vet. Bras. 33:724730 .

Nunes A.L. 2013. Probiótico na dieta de surubins Pseudoplatystoma spp. Dissertação de Mestrado em Zootecnia, Universidade Estadual de Mato Grosso do Sul, Aquidauana, MS. 99p.

Otutumi L.K. 2006. Uso de probiótico para codornas de corte (Coturnix coturnix sp.). Tese de Doutorado em Zootecnia, Universidade Estadual de Maringá, Maringá, PR. 73p.

Pelicano E.R.L., Souza P.A., Souza H.B.A., Oba A., Norkus C.E.A., Kodawara L.M. \& Lima T.A. 2003. Morfometria e ultra-estrutura da mucosa intestinal de frangos de corte alimentados com dietas contendo diferentes probióticos. Revta Port. Ciênc. Vet. 98:125-134.

Prophet E.B., Mills B., Arrington J.B. \& Sobin L.H. 1992. Laboratory Methods in Histotechnology. American Registry of Pathology, Armed Forces Institute of Pathology, Washington, DC. 279p.

Rahiman K.M.M., Jesmim Y., Thomas A.P. \& Hatha A.A.M. 2010. Probiotic effect of Bacillus NL110 and Vibrio NE17 on the survival, growth performance and immune response of Macrobrachium rosenbergii (de Man). Aquac. Res. 41:120-134.

Rotta M.A. 2003. Aspectos Gerais da Fisiologia e Estrutura do Sistema Digestivo dos Peixes Relacionados à Piscicultura. Embrapa Pantanal, Corumbá, MS. 48p.

Rust M.B. 2002. Nutritional physiology, p.367-446. In: Halver J. E. \& Hardy R.W. (Eds), Fish Nutrition. Editora Academic Press, SanDiego, California.

Saarela M., Mogensen G., Fondén R., Matto J. \& Mattila-Sandholm T. 2000. Probiotic bacteria safety, functional and technological properties. J. Biotech. 8:197-215.

Salze G., McLean E., Schwarz M.H. \& Craig S.R. 2008. Dietary mannanoligosaccharide enhances salinity tolerance and gut development of larval cobia. Aquaculture 274:148-152.

Silva L.P. \& Nörnberg J.L. 2003. Prebióticos na nutrição de não ruminantes. Ciência Rural 33:983- 990.

Schwarz K.K., Furuya W.M., Natall M.R.M., Gaudez M.C. \& Lima P.A.G. 2011. Mananoligossacarídeo em dietas para larvas de tilápia. R. Bras. Zootec. 40(12):2634-2640.

Urbinati E.C. \& Carneiro P.C.F. 2004. Práticas de Manejo e estresse dos peixes em piscicultura intensiva, p.171-193. In: Cyrino J.E.P., Urbinati E.C. \& Castagnolli N. (Eds.). Tópicos Especiais em Piscicultura Tropical. Editora TecArt, São Paulo.

Verschuere L., Rombaut G., Sorgeloos P. \& Verstraete W. 2000. Probiotic bacteria as biological control agents in aquaculture. Microbiol. Mol. Biol. Rev. 64:655-671.

Yilmaz E., Genc M.A. \& Genc E. 2007. Effects of dietary mannan oligosaccharides on growth, body composition, and intestine and liver histology of rainbow trout, Oncorhynchus mykiss. Isr. J. Aquac. Bamidgeh. 59:182188. 